\title{
Characterization of multilayer self-organized InAs quantum dot embedded waveguides at 1.3 and $1.5 \mu \mathrm{m}$
}

\author{
B. I. Akca \\ Material Science and Nanotechnology Department, Bilkent University, Ankara, Turkey \\ A. Dana, A. Aydinli \\ Physics Department, Bilkent University, Ankara, Turkey \\ M. Rossetti, L. Li, A. Fiore \\ Institute of Quantum Electronics and Photonics, Ecole Polytechnique Fédérale de Lausanne EPFL, \\ CH-1015 Lausanne, Switzerland \\ N. Dagli \\ ECE Department, University of California at Santa Barbara, California, USA
}

\begin{abstract}
Semiconductor-based nonlinear optical and electro-optic materials would be useful for switching and modulation devices in photonic integrated circuits. Due to the modification in the density of states, quantum dots and quantum wells are expected to exhibit enhanced optical nonlinearities and enhanced electro-optic effects [1]. In this regard, we characterized the electro-optic coefficient and loss of multilayer InAs quantum dot laser structures at 1309 and $1515 \mathrm{~nm}$. Quantum dot waveguides were grown by molecular beam epitaxy, where the active region is formed by three or five layers of self-assembled InAs QDs, covered by a $5-\mathrm{nm} \mathrm{In}_{0.15} \mathrm{Ga}_{0.85} \mathrm{As}$ QW and separated from each other by a $40-\mathrm{nm}$ GaAs spacer layer. The areal dot density of the lens-shaped QDs is $3 \times 10^{10} \mathrm{~cm}^{-2}$. The laser cavity is clad by $1.5 \mu \mathrm{m}$ of $\mathrm{Al}_{0.7} \mathrm{Ga}_{0.3} \mathrm{As}$, which is $n$-doped on the substrate side and $p$-doped on the top side. Devices are ridge-waveguide lasers with 8 and $15 \mu \mathrm{m}$ ridge widths and lasing is peaked at around 1285nm [2]. Measurement of the electro-optic coefficients away from EL emission peak was carried out by coupling $1.5 \mu \mathrm{m}$ light from a tunable laser (Santec Tunable LD Light Source TSL-520) onto one end of the waveguide with a lensed fiber. A controlled DC voltage source was used to apply 0 to 6 Volts reverse bias to the samples. Transmission through the device was recorded as a function of wavelength. The index of refraction was inferred from the Fabry-Perot cavity response and the linear electro-optic coefficients $\left(\mathrm{r}_{41}\right)$ are obtained by fitting the measured data to the equation of transmission through a Fabry Perot modulator and calculating the refractive index difference between two specific voltages. The average values for electro-optic coefficients $\left(\mathrm{r}_{41} / \Gamma\right)$ of 3QDs and 5QDs samples are given as $7.7 \times 10^{-13} \mathrm{~m} / \mathrm{V}$ and $17 \times 10^{-13} \mathrm{~m} / \mathrm{V}$. Loss characterization were carried out by using a $1.3 \mu \mathrm{m}$ light from a thermally tunable laser. Transmission through the device was recorded as a function of wavelength. Loss coefficient is found to be wavelength and bias voltage dependent. At $1.3 \mu \mathrm{m}$, typical loss differences are $\sim 3 \mathrm{~dB} / \mathrm{cm}$ for a bias voltage difference of $5 \mathrm{~V}$ for the 3 QDs sample and $\sim 4 \mathrm{~dB} / \mathrm{cm}$ for a bias voltage difference of $4 \mathrm{~V}$ for the 5QDs sample.
\end{abstract}

References:

[1]. L. Davis, K. Ko, W. Li, H.Sun, Y. Lam, T. Brock, S. Pang, and P. Bhattacharya, "Photoluminescence and electro-optic properties (25-35 nm diameter) quantum boxes", Appl. Phys. Lett., vol. 62, No.22, May 1993

[2]. A. Markus, J. X. Chen, C. Paranthoen, and A. Fiore," Simultaneous two-state lasing in quantum-dot lasers," Applied Physics Letter, vol. 82, No. 12, March 2003. 